\title{
Effect of phenotypic residual feed intake and diet type on ruminal microbial population in beef heifers
}

\author{
C A Carberry ${ }^{1,2}$, D A Kenny ${ }^{2}$, M S McCabe $^{1}$, S M Waters $^{1}$
}

${ }^{1}$ Teagasc Animal Bioscience Centre, Grange, Dunsany, Co. Meath, Ireland, ${ }^{2}$ School of Agriculture,Food and veterinary medicine, University college Dublin, Belfield, Dublin 4, Ireland

Email: ciara.carberry@teagasc.ie

Introduction In Ireland, methane $\left(\mathrm{CH}_{4}\right)$ emissions from ruminants accounts for approximately $51 \%$ of greenhouse gas emissions from the agriculture sector (McGettigan et al., 2008). Additionally, $\mathrm{CH}_{4}$, formed as a by-product of rumen microbial fermentation can account for up to $12 \%$ of dietary gross energy. Therefore reducing enteric $\mathrm{CH}_{4}$ production may also improve feed efficiency. Despite its importance as a significant contributor to global warming, relatively little is known about the microbial population in the rumen. Selection for improved feed efficiency in beef cattle, measured as residual feed intake (RFI), has been shown to reduce total and feed intake corrected $\mathrm{CH}_{4}$ emissions without compromising animal production (Hegarty et al., 2007). However, little is known about the biological mechanisms controlling this effect. The ruminal microbial ecosystem is comprised of diverse symbiotic populations of anaerobic bacteria, archaea, ciliated protozoa and fungi, though there is a dearth of published information on how these microorganisms are influenced by either intrinsic or extrinsic factors. Quantification of these microbial populations and assessment of how they differ across different animals and diet types will increase our understanding of host-microbial interactions in ruminants.

Materials and methods Limousin x Friesian beef heifers $(n=86)$, initially selected on the basis of sire EBV for RFI, were ranked on the basis of phenotypic RFI, calculated over an 80-day period while consuming a 30:70 maize silage:concentrate TMR diet (on a DM basis). The seven highest (HRFI; least efficient) and seven lowest (LRFI; most efficient) ranking animals were selected for use in this study. Both groups had similar mean bodyweight and ADG at ranking but HRFI had, on average, 20\% higher DMI. Following ranking on RFI all animals were allocated to a grass silage diet for six weeks (Period 1). Three months later all animals were again offered a 30:70 maize silage:concentrate TMR over a six week period (Period 2). Both silage and TMR diets were offered ad libitum. Ruminal fluid was sampled at the end of each period using a specialised trans-oesophageal sampling device. Total microbial DNA was isolated from the ruminal fluid using a repeated bead beating method. A qPCR SYBR Green assay was developed to quantify key microbial groups using PCR primers to target DNA sequences of total rumen bacteria, methanogens, Ruminococcus flavefaciens, Fibrobacter succinogenes and protozoa. Abundance of these microbes was expressed as a proportion of total estimated rumen bacterial $16 \mathrm{~S}$ rDNA according to the equation: relative quantification $=2^{-(\mathrm{Ct} \text { target-Ct total bacteria) }}$, where $\mathrm{Ct}$ represents threshold cycle. Data were analysed using mixed models ANOVA (PROC MIXED, SAS 2006). The final model included terms for RFI group, diet and their interaction, with differences between means statistically significant at $P<0.05$.

Results No RFI phenotype $\mathrm{x}$ diet interactions for any of the microbial populations measured were observed. The effect of phenotypic RFI and diet on key microbial populations is shown in Table 1 . There was no effect $(P>0.05)$ of RFI phenotype on the quantity of any microbial species measured. However, dietary period affected $(P<0.05)$ ruminal microbial populations manifested as a reduction in methanogen, Ruminococcus flavefaciens, Fibrobacter succinogens numbers and an increase in protozoa numbers between periods 1 and 2 .

Table 1 Effect of phenotypic RFI and diet on ruminal microbial populations ${ }^{1,2}$

\begin{tabular}{|c|c|c|c|c|c|c|c|c|c|}
\hline & \multicolumn{2}{|c|}{ RFI } & \multirow[t]{2}{*}{ SED } & \multicolumn{2}{|c|}{ Diet } & \multirow[t]{2}{*}{ SED } & \multicolumn{3}{|c|}{ Significance $^{3}$} \\
\hline & $\mathrm{H}$ & $\mathrm{L}$ & & GS & TMR & & RFI & Diet & $\mathrm{RxD}$ \\
\hline Methanogens & 4.04 & 4.84 & 0.985 & 6.15 & 2.74 & 0.985 & NS & $* * *$ & NS \\
\hline Ruminococcus flavefacien ${ }^{4}$ & 0.13 & 0.05 & 0.050 & 0.17 & 0.015 & 0.050 & NS & $* * *$ & NS \\
\hline Fibrobacter succinogenes ${ }^{4}$ & 2.21 & 1.59 & 0.661 & 3.34 & 0.46 & 0.661 & NS & $* * *$ & NS \\
\hline Protozoa & 0.54 & 0.73 & 0.209 & 0.57 & 0.70 & 0.209 & NS & $*$ & NS \\
\hline
\end{tabular}

$\mathrm{R}=\mathrm{RFI}$; $\mathrm{D}=$ Diet. NS=Non-significant $(P>0.05) .{ }^{*} P<0.05, * * P<0.01, * * * P<0.001 .{ }^{1}$ Microbes measured as a proportion of total estimated rumen bacterial $16 \mathrm{~S}$ rDNA, relative quantification $=2^{-(\mathrm{Ct} \text { target-Ct total bacteria) }} \times 10^{3}$. ${ }^{2}$ Data corrected for DMI. ${ }^{3}$ Significance values for transformed data. Back transformed means presented for clarity. ${ }^{4}$ Cellulolytic bacteria

Conclusions There was no clear evidence from the current study of differences in inherent ruminal microbial population between animals ranked as either efficient or inefficient for feed energy utilisation. However, it was clear that diet can influence the number of methanogenic and cellulolytic microbes with reductions observed in these when animals were offered the high starch, high energy diet. These results are consistent with lower ruminal $\mathrm{CH}_{4}$ emissions recorded on this TMR diet (McDonnell et al., 2009). Dennis et al., 1983 reported increased protozoa numbers in cattle as the proportion of concentrate in the diet increased, as found in the present study. Further investigation is warranted to determine the effect of animal RFI phenotype and diet on the abundance of other important ruminal microorganisms to improve our understanding of feed efficiency and methanogenesis in cattle and how these processes might be influenced by diet type.

Acknowledgements The authors gratefully acknowledge funding provided under the National Development Plan, through the Research Stimulus Fund administered by the Department of Agriculture, Fisheries \& Food, Ireland. RSF 05-224.

References Dennis S.M., Arambel M.J, Bartley E.E. and Dayton A.D. 1983. Journal of Dairy Science 66, $1248-1254$. Hegarty R.S., Goopy J.S., Herd R.M. and McCorkell B. 2007. Journal of Animal Science 85, 1479-1486.

McGettigan M., Duffy P., Hyde B. and O’Brien P. 2008. Ireland National Invetory Report 2008.

McDonnell R., Hart K.J., Boland T.M. and Kelly A.K 2009. SAS.2006. SAS Institute. Cary, NC, USA. 